\title{
13. PETROGRAPHIC AND GEOCHEMICAL PROPERTIES OF TEPHRAS IN DEEP SEA DRILLING PROJECT CORES FROM THE NORTH PHILIPPINE SEA
}

\author{
Toshio Furuta, Ocean Research Institute, University of Tokyo, \\ and \\ Fusao Arai, Department of Geology, Gunma University, Maebashi, Japan
}

\begin{abstract}
Refractive index and chemical composition were determined for glass shards contained in more than 100 tephra layers in DSDP Leg 58 sediment cores collected in the Shikoku Basin, North Philippine Sea. The refractive index is consistent with chemical composition. Refractive index and total iron show a linear relationship. Tephra in Pleistocene and Pliocene sediments is mostly rhyolitic and dacitic (non-alkali), whereas tephra in the Miocene shows wide composition variations in the eastern part of the basin. Basaltic tephra is recognized in Miocene sediments at Sites 443 and 444 , but not at Site 442 , west of the other two sites. This indicates that the basaltic tephra came from eruption relatively close to those drill sites (perhaps the Kinan Seamounts and the Shichito-Iwo Jima volcanic arc), although the exact source has not been identified.
\end{abstract}

\section{INTRODUCTION}

Recent mineralogical and chronological studies of deep-sea tephra layers in DSDP and piston cores have enabled us to consider the geological and geophysical problems related to volcanism in and around island arcs and oceanic islands.

During DSDP Leg 58, three sites were drilled in the central part of the Shikoku Basin (Figure 1). Abundant tephra layers in deep-sea sediments were identified clearly in sediments from the lower Miocene to Holocene. Volcanic glass shards in these tephra layers are heterogeneous in chemical composition, as determined by electron-microprobe analyses and measurement of refractive index, so that correlation between these tephra layers and their sources is possible.

DSDP tephra layers and their correlation with subaerial volcanic materials have been investigated extensively (Jones, 1973; Scheidegger and Kulm, 1975; Scheidegger et al., 1978; Ninkovich, 1979; Fujioka et al., in press; Furuta and Arai, in press). Scheidegger et al. (1978), using data from 33 analyzed tephra layers of the Pleistocene and Pliocene at DSDP Site 192 in the northwestern Pacific, demonstrated that glass shards have been subjected to progressive hydration, with minor exchanges of $\mathrm{K}_{2} \mathrm{O}, \mathrm{MgO}$, and $\mathrm{CaO}$. They also suggested that hydration occurs rapidly and leads to a uniform water content of 4.5 to 5.0 per cent during exposure to seawater over several hundred thousand years. Scheidegger and Kulm (1975), using chemical composition of glass shards, showed that the tephra layers at DSDP Site 178 in the Gulf of Alaska correlate with the volcanic activities of the Aleutian and Alaska arcs. Ninkovich (1979) analyzed tephra layers of deep-sea piston and DSDP cores from the northeastern Indian Ocean and suggested that three distinct provinces can be recognized from chemical data. He also demonstrated that hydration in glass shards reaches a "saturation point" of 4 to 5 per cent in 250,000 to 400,000 years.

This paper describes petrographic and geochemical properties of tephra layers from the Shikoku Basin. We do not attempt to discuss the petrogenetic implications of the analyzed data, although we do give some idea of the sources of tephra. We present results of microprobe analyses of glass shards and constituent minerals in $\mathbf{3 2}$

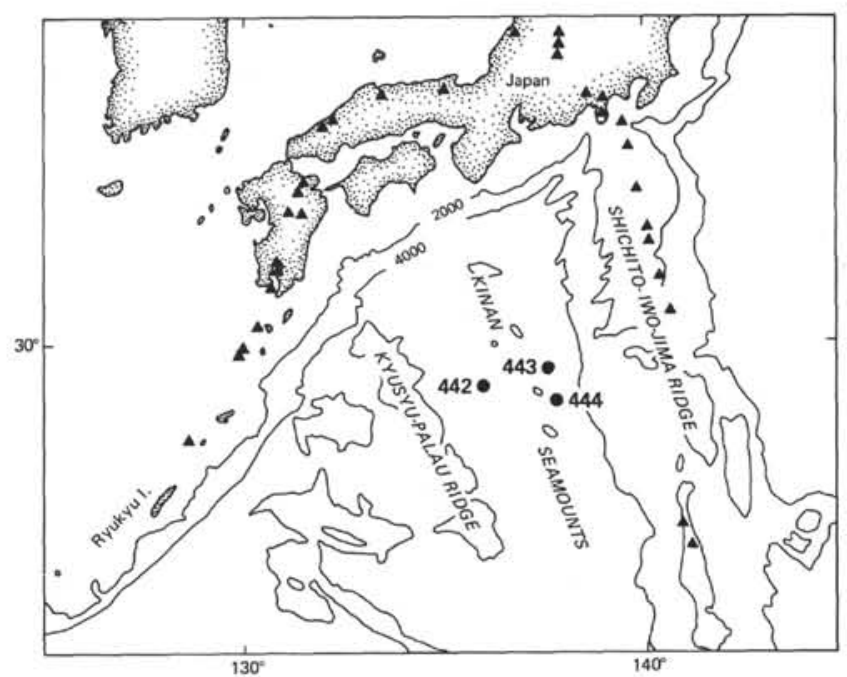

Figure 1. Location of Leg 58 drill sites in the Shikoku Basin and Quaternary volcanoes (triangles). Bathymetry in meters. 
tephra layers from the early Miocene to Holocene and measurements of refractive index of glass shards in all tephra layers.

It is worth emphasizing that at two sites (443 and 444), tephra of basaltic composition was recovered from Miocene sediments. Tephra found so far in DSDP cores has been mostly rhyolitic to andesitic; it is seldom that basaltic tephra, such as in the Shikoku Basin, has been recognized. Therefore, examination of these tephra layers is of great interest from the viewpoint of the sedimentary environments and tectonic developments of the Shikoku Basin.

Twenty-three tephra layers of late-Eocene to Holocene age were also sampled at Site 445, at the crest of the Daito Ridge. However, only petrographic characters are presented (Appendix, this chapter), because their features have not been examined in detail.

\section{PETROGRAPHIC FEATURES}

Tephra layers in deep-sea sediments of the Shikoku Basin range from early-Miocene to Holocene in age. Twenty layers from Site 442 (Holes 442A and 442B), 30 layers from Site 443, and 60 layers from Site 444 (Holes 444 and 444A) were investigated. Table 1 shows the numbers of tephra layers from the three sites. Techniques used for preparation of tephra samples for refractive index and chemical analysis are the same as described by Furuta and Arai (in press).

Refractive indexes of tephra layers are shown in Table 2 and Figure 2. Volcanic glass shards are classified as pumice, bubble-wall, and scoria types by their shapes. Plate 1 illustrates typical examples.

At Site 442 , refractive indexes of glass shards in early-Miocene to Holocene tephra layers range from 1.498 to 1.512 . The glass is colorless, with the exception of one layer at $442-3-6,48-50 \mathrm{~cm}$. Glass shards from thiś site are pumice and (or) bubble-wall type (Table 2). The refractive index for the layer at $442-3-6,48-50 \mathrm{~cm}$ is somewhat higher than that of shards from other layers, with values from 1.518 to 1.521 .

At Site 443 , approximately $100 \mathrm{~km}$ east of Site 442 , refractive indexes of glass shards show a wide range, from 1.499 to 1.599 . Basaltic tephra predominates in late-Miocene sediments. Glass shards at this site are pumice and (or) bubble-wall and scoria types. The first and second types have relatively low refractive indexes, while the third has high indexes. Glass shards with relatively low refractive indexes are colorless, and those with high indexes are brown. At Site $444,70 \mathrm{~km}$ south-

TABLE 1

Numbers of Tephra Layers Cored at the Shikoku Basin Sites

\begin{tabular}{lccc}
\hline \multicolumn{1}{c}{ Age } & Site 442 & Site 443 & Site 444 \\
\hline Pleistocene & 11 & 4 & 12 \\
Pliocene & 2 & 3 & 13 \\
Miocene & 7 & 23 & 35 \\
Total & 20 & 30 & 60 \\
\hline
\end{tabular}

east of Site 443, petrographic features and refractive indexes of glass shards appear to be similar to those for Site 443 tephra layers.

At this site, the highest refractive indexes, 1.600 to 1.610 , are found at $444 \mathrm{~A}-10-1,92-94 \mathrm{~cm}$. Refractive index and color of glass shards seem to be controlled by the abundance of transition metals $\left(\mathrm{FeO}, \mathrm{TiO}_{2}, \mathrm{MnO}\right.$, etc.).

\section{CHEMICAL COMPOSITION OF VOLCANIC GLASS}

Five samples from the Site 442 tephra layers, 16 from Site 443 , and 11 from Site 444 were chemically analyzed by electron microprobe. The results are shown in Table 3. $\mathrm{SiO}_{2}$ content ranges from 47 to 74 per cent, corresponding to compositions from basalt to rhyolite. $\mathrm{Al}_{2} \mathrm{O}_{3}$ content varies from 11 to 16 per cent, values consistent with those reported for normal volcanic rocks with the same $\mathrm{SiO}_{2}$ contents. $\mathrm{FeO}$ (total iron as $\mathrm{FeO}$ ) varies from 0.9 to 13 per cent. This variation is mainly responsible for the changes in refractive index of glass shards. $\mathrm{MgO}$ content also varies considerably and is inversely related to $\mathrm{SiO}_{2}$ content. $\mathrm{CaO}, \mathrm{Na}_{2} \mathrm{O}$, and $\mathrm{K}_{2} \mathrm{O}$ contents vary considerably. Such variation in $\mathrm{CaO}$ and alkalis can be explained by magmatic differentiation, as well as by hydration of glasses after deposition. In particular, the alkali contents are likely to vary significantly as a result of hydration (Aramaki and Lipman, 1965; Aramaki and Haramura, 1966; Jezek and Noble, 1978).

In order to test whether the analyzed glass shards belong to alkali or non-alkali rock series, total alkalis are plotted against $\mathrm{SiO}_{2}$ (Figure 3) (Kuno, 1965). In this diagram, data were recalculated on a water-free basis. Most values from Pleistocene and Pliocene glass shards are fitted to the non-alkali rock series (Figure 3a), but data from Miocene glass shards are scattered (Figure $3 b)$. Miocene glass shards from three tephra layers at Site 444 with low $\mathrm{SiO}_{2}$ contents, and three from Site 443 with high $\mathrm{SiO}_{2}$ contents, belong to the alkali rock series. This suggests that two different types of volcanic activity occurred during the same time in the Shikoku Basin or adjacent areas.

In the upper Miocene, different rock series, alkalic and non-alkalic, are represented by the deep-sea tephra layers. Figure 3 shows that the compositions of glass shards from different sites are well clustered. Such similarity of chemical composition is very useful for correlation of tephra layers.

Data from each tephra layer are plotted in an AFM diagram in Figure 4. This diagram indicates the fractionation evidenced by glass-shard compositions. The Miocene tephra layers show patterns of high iron concentration, typical of the tholeitic fractional crystallization trends, with three exceptions (Figure $4 b$ ); these are from Miocene tephra layers at Site 444 and belong to the alkali rock series. These samples have a high alkali content $(4-5 \%)$, in spite of an $\mathrm{SiO}_{2}$ content lower than 50 per cent and are very similar to typical alkali olivine basalt. From Figures 3 and 4, it is inferred that tephra layers from the Shikoku Basin originated from at least two different volcanic activities. 
TABLE 2

Petrographic Characters of Tephra Layers

\begin{tabular}{|c|c|c|c|c|c|c|c|c|c|}
\hline Age & $\begin{array}{c}\text { Sample } \\
\text { (interval in } \mathrm{cm} \text { ) }\end{array}$ & $\begin{array}{l}\text { Sub- } \\
\text { bottom } \\
\text { Depth } \\
\text { (m) }\end{array}$ & Constituent Minerals ${ }^{a}$ & Type ${ }^{b}$ & Color & $\begin{array}{l}\text { Size } \\
(\mathrm{mm})\end{array}$ & canic Glass & Mean Range & Mode \\
\hline $\begin{array}{l}\stackrel{0}{0} \\
\stackrel{0}{0} \\
\frac{.0}{2} \\
\frac{0}{a}\end{array}$ & $\begin{array}{c}442 \mathrm{~A}-2-5,100-102 \mathrm{c} \\
3-3,66-68^{\mathrm{c}} \\
3-6,48-50^{\mathrm{c}} \\
5-3,30-32 \mathrm{c} \\
5-3,132-134 \\
5-4,75-77 \\
5-5,104-106 \\
7-1,98-100 \mathrm{c} \\
7-4,48-50 \\
10-4,31-33 \\
13-2,80-82\end{array}$ & $\begin{array}{r}16.00 \\
22.66 \\
26.98 \\
41.30 \\
42.32 \\
43.25 \\
45.04 \\
57.98 \\
61.98 \\
90.31 \\
116.30\end{array}$ & $\begin{array}{l}\mathrm{pl}++, \mathrm{hb}+ \\
\mathrm{pl}++, \mathrm{hb}++, \mathrm{hy}+, \mathrm{op}+ \\
\mathrm{pl}++, \mathrm{hy}+, \mathrm{au}+, \mathrm{op}+ \\
\mathrm{pl}+, \mathrm{hb}+ \\
\mathrm{pl}+, \mathrm{hb}+ \\
\mathrm{pl}+, \mathrm{hb}+ \\
\mathrm{pl}+, \mathrm{op}+ \\
\mathrm{pl}+, \mathrm{hb}+ \\
\mathrm{pl}+, \mathrm{hb}+, \mathrm{bi}+, \mathrm{px}+ \\
\mathrm{pl}+, \mathrm{op}+ \\
\mathrm{pl}++, \mathrm{hy}+, \mathrm{au}+, \mathrm{ho}+\end{array}$ & $\begin{array}{l}\mathrm{pm} \simeq \mathrm{bw} \\
\mathrm{pm} \simeq \mathrm{bw} \\
\mathrm{pm} \\
\mathrm{pm}<\mathrm{bw} \\
\mathrm{pm} \simeq \mathrm{bw} \\
\mathrm{pm} \simeq \mathrm{bw} \\
\mathrm{pm}>\mathrm{bw} \\
\mathrm{pm} \simeq \mathrm{bw} \\
\mathrm{pm} \simeq \mathrm{bw} \\
\mathrm{pm}>\mathrm{bw} \\
\mathrm{pm} \simeq \mathrm{bw}\end{array}$ & $\begin{array}{l}\text { clear } \\
\text { clear } \\
\text { p. brown } \\
\text { clear } \\
\text { clear } \\
\text { clear } \\
\text { clear } \\
\text { clear } \\
\text { clear } \\
\text { clear } \\
\text { clear }\end{array}$ & $\begin{array}{l}0.3 \\
0.4 \\
0.6 \\
0.2 \\
0.3 \\
0.3 \\
0.2 \\
0.3 \\
0.3 \\
0.3 \\
0.4\end{array}$ & $\begin{array}{l}1.499-1.501 \\
1.500-1.502 \\
1.517-1.522 \\
1.500-1.505 \\
1.500-1.502 \\
1.500-1.505 \\
1.499-1.501 \\
1.501-1.503 \\
1.500-1.503 \\
1.504-1.509 \\
1.500-1.502\end{array}$ & $\begin{array}{l}1.500-1.501 \\
1.518-1.521 \\
1.501-1.504 \\
1.500-1.503\end{array}$ & $\begin{array}{l}1.501 \\
1.4995 \\
1.502 \\
1.507 \\
1.501\end{array}$ \\
\hline 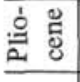 & $\begin{array}{l}20-1,25-27 \\
21-3,84-86 \\
\end{array}$ & $\begin{array}{l}180.75 \\
193.84 \\
\end{array}$ & $\begin{array}{l}\mathrm{pl}+, \mathrm{op}+ \\
\mathrm{pl}++, \mathrm{bi}++\end{array}$ & $\begin{array}{l}\mathrm{pm}>\mathrm{bw} \\
\mathrm{pm}>\mathrm{bw}\end{array}$ & $\begin{array}{l}\text { clear } \\
\text { clear }\end{array}$ & $\begin{array}{l}0.2 \\
0.4 \\
\end{array}$ & $\begin{array}{l}1.503-1.505 \\
1.498-1.500 \\
\end{array}$ & & $\begin{array}{l}1.504 \\
1.499 \\
\end{array}$ \\
\hline 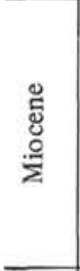 & $\begin{array}{c}28-3,117-119 \\
29-1,145-147 \\
29-2,66-68 \\
442 \mathrm{~B}-1-1,70-72 \\
1-2,96-98 \\
1-2,134-136 \\
1, \mathrm{CC}\end{array}$ & $\begin{array}{l}260.67 \\
267.45 \\
268.16 \\
268.20 \\
269.96 \\
270.34 \\
270.80\end{array}$ & $\begin{array}{l}\mathrm{pl}+, \mathrm{bi}+ \\
\mathrm{pl}+, \mathrm{op}+ \\
\mathrm{pl}++, \mathrm{qt}+, \mathrm{bi}+, \mathrm{hb}+ \\
\mathrm{pl}+, \mathrm{hy}+, \mathrm{au}+ \\
\mathrm{pl}+, \mathrm{op}+ \\
\mathrm{hb}+, \mathrm{au}+, \mathrm{bi}+\end{array}$ & $\begin{array}{l}\mathrm{pm} \simeq \mathrm{bw} \\
\mathrm{pm} \simeq \mathrm{bw} \\
\mathrm{pm} \simeq \mathrm{bw} \\
\mathrm{pm}<\mathrm{bw} \\
\mathrm{bw} \\
\mathrm{pm}<\mathrm{bw} \\
\mathrm{pm} \simeq \mathrm{bw}\end{array}$ & $\begin{array}{l}\text { clear } \\
\text { clear } \\
\text { clear } \\
\text { clear }\end{array}$ & $\begin{array}{l}0.3 \\
0.1 \\
0.4 \\
\\
0.5 \\
0.4 \\
0.3 \\
0.5 \\
\end{array}$ & $\begin{array}{l}1.500-1.501 \\
1.500-1.501 \\
1.500-1.504 \\
1.504-1.508 \\
1.504-1.511 \\
1.508-1.512 \\
1.500-1.503 \\
\end{array}$ & $\begin{array}{l}1.500-1.503 \\
1.509-1.511 \\
1.500-1.501 \\
\end{array}$ & 1.506 \\
\hline 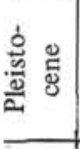 & $\begin{array}{c}443-1-4,64-66^{c} \\
4-2,80-82^{c} \\
8-5,26-28 \mathrm{c} \\
11-5,55-57 \mathrm{c} \\
\end{array}$ & $\begin{array}{r}5.14 \\
33.44 \\
70.26 \\
99.05 \\
\end{array}$ & $\begin{array}{l}\mathrm{pl}+\text {, hy }+, \mathrm{au}+, \text { op }+ \\
\mathrm{pl}+, \mathrm{hy}+, \mathrm{au}+, \text { op }+ \\
\mathrm{pl}++, \text { op }+ \\
\mathrm{pl}+\end{array}$ & $\begin{array}{l}\mathrm{pm} \& \mathrm{bw} \\
\mathrm{pm} \geqslant \mathrm{bw} \\
\mathrm{pm}>\mathrm{bw} \\
\mathrm{pm}>\mathrm{bw}\end{array}$ & $\begin{array}{l}\text { clear } \\
\text { p. brown } \\
\text { clear } \\
\text { clear } \\
\end{array}$ & $\begin{array}{l}0.2 \\
0.3 \\
0.2 \\
0.3\end{array}$ & $\begin{array}{l}1.499-1.501 \\
1.518-1.525 \\
1.499-1.501 \\
1.500-1.503\end{array}$ & $1.519-1.522$ & $\begin{array}{l}1.500 \\
1.520 \\
1.500 \\
1.502 \\
\end{array}$ \\
\hline$\stackrel{1}{\dddot{0}}$ & $\begin{array}{l}18-1,118-121 \\
18-3,110-112 \\
22-2,139-141^{c}\end{array}$ & $\begin{array}{l}160.18 \\
163.10 \\
199.89\end{array}$ & $\begin{array}{l}\text { ho }++, \mathrm{pl}+\text {, hy }+ \\
\mathrm{pl}+\text {, hy }+, \text { au }+ \text {, op }+ \\
\mathrm{pl}+\text {, hy }+ \text {, ho }+ \text {, op }+\end{array}$ & $\begin{array}{l}\mathrm{pm}>\mathrm{bw} \\
\mathrm{pm}>\mathrm{bw} \\
\mathrm{pm}>\mathrm{bw}\end{array}$ & $\begin{array}{l}\text { clear } \\
\text { clear } \\
\text { clear }\end{array}$ & $\begin{array}{l}0.3 \\
0.2 \\
0.3\end{array}$ & $\begin{array}{l}1.504-1.508 \\
1.503-1.506 \\
1.504-1.510 \\
\end{array}$ & $\begin{array}{l}1.504-1.507 \\
1.505-1.508\end{array}$ & $\begin{array}{l}1.505 \\
1.505\end{array}$ \\
\hline $\begin{array}{l}\stackrel{\Xi}{8} \\
\stackrel{\varrho}{\Sigma}\end{array}$ & $\begin{array}{l}31-3,29-31^{\mathrm{c}} \\
31-4,6-8 \mathrm{c} \\
32-1,106-108^{\mathrm{c}} \\
32-2,105-107 \\
34-2,43-45 \\
39-2,69-71^{\mathrm{c}} \\
40-2,22-24 \mathrm{c} \\
40-2,139-140^{\mathrm{c}} \\
42-1,22-24 \\
42-1,92-94 \\
43-1,30-32 \\
43-1,69-71 \\
43-2,22-24 \\
43-2,36-38 \\
45-1,92-94 \mathrm{c} \\
45-1,106-108 \mathrm{c} \\
45, \mathrm{CC} \\
46-1,98-99 \\
46-1,106-108 \\
46-1,111-113 \\
46-2,181-183^{\mathrm{c}} \\
48-1,85-87 \mathrm{c} \\
49-1,76-78\end{array}$ & $\begin{array}{l}285.79 \\
287.06 \\
293.06 \\
304.05 \\
312.93 \\
360.69 \\
369.72 \\
370.89 \\
387.22 \\
387.92 \\
396.80 \\
397.19 \\
398.22 \\
398.36 \\
416.12 \\
416.56 \\
417.28 \\
425.98 \\
426.06 \\
426.11 \\
428.31 \\
444.85 \\
454.26\end{array}$ & $\begin{array}{l}\mathrm{pl}++, \mathrm{au}+, \mathrm{ol}+ \\
\mathrm{pl}+ \\
\mathrm{pl}++ \\
\mathrm{pl}+, \mathrm{op}+ \\
\mathrm{pl}+ \\
\mathrm{pl}+, \mathrm{hy}+ \\
\mathrm{pl}+ \\
\mathrm{pl}+, \mathrm{hy}+ \\
\mathrm{pl}+ \\
\mathrm{pl}++, \mathrm{hy}+ \\
\mathrm{pl}++, \mathrm{bi}+ \\
\mathrm{pl}+, \mathrm{au}+ \\
\mathrm{pl}+ \\
\mathrm{pl}++, \mathrm{hy}+ \\
\mathrm{pl}+, \mathrm{au}+ \\
\mathrm{pl}+, \mathrm{au}+ \\
\mathrm{au}++, \mathrm{hy}+, \mathrm{op}+\end{array}$ & $\begin{array}{l}\text { sco } \\
\text { sco } \\
\text { sco } \\
\text { pm }>\text { bw } \\
\text { sco } \\
\text { pm } \\
\text { pm } \\
\text { pm } \simeq \text { bw } \\
\text { sco } \\
\text { pm }<\text { bw } \\
\text { pm } \\
\text { pm }<\text { bw } \\
\text { pm }>\text { bw } \\
\text { pm } \\
\text { pm }<\text { bw } \\
\text { pm }>\text { bw } \\
\text { pm } \geqslant \text { bw } \\
\text { pm } \simeq \text { bw } \\
\text { pm }>\text { bw } \\
\text { pm }>\text { bw } \\
\text { sco } \\
\text { pm }>\text { bw } \\
\text { pm }>\text { bw }\end{array}$ & $\begin{array}{l}\text { brown } \\
\text { brown } \\
\text { brown } \\
\text { clear } \\
\text { brown } \\
\text { clear? } \\
\text { clear } \\
\text { brown } \\
\text { clear } \\
\text { clear } \\
\text { clear } \\
\text { clear } \\
\text { p. brown } \\
\text { p. brown } \\
\text { p. brown } \\
\text { p. brown } \\
\text { clear } \\
\text { clear } \\
\text { clear } \\
\text { brown } \\
\text { p. brown } \\
\text { clear }\end{array}$ & $\begin{array}{l}0.5 \\
0.3 \\
0.3 \\
0.1 \\
\\
0.4 \\
1.0 \\
0.2 \\
0.2 \\
0.5 \\
1.0 \\
0.3 \\
0.5 \\
0.4 \\
0.4 \\
0.2 \\
0.3 \\
0.2 \\
0.3 \\
0.3 \\
0.3 \\
0.4 \\
0.2 \\
\end{array}$ & $\begin{array}{l}1.580-1.592 \\
1.584-1.592 \\
1.583-1.595 \\
1.502-1.505 \\
1.583-1.588 \\
1.519-1.525 \\
1.538-1.546 \\
1.507-1.510 \\
1.592-1.599 \\
1.499-1.500 \\
1.505-1.508 \\
1.500-1.502 \\
1.501-1.506 \\
1.528-1.547 \\
1.507-1.513 \\
1.530-1.543 \\
1.518-1.550 \\
1.503-1.515 \\
1.503-1.505 \\
1.501-1.555 \\
1.582-1.590 \\
1.527-1.533 \\
1.508-1.520\end{array}$ & $1.501-1.505$ & $\begin{array}{l}1.522 \\
1.508 \\
1.4995 \\
1.506 \\
1.501 \\
1.503 \\
1.510\end{array}$ \\
\hline $\begin{array}{l}\frac{2}{8} \\
\frac{0}{0} \\
\frac{0}{2} \\
\frac{0}{2}\end{array}$ & $\begin{array}{l}444-2-1,10-12 \\
2-1,80-82 \\
2-2,116-118 \\
2-3,112-114 \\
4-5,54-56 \mathrm{c} \\
4-5,106-108^{\mathrm{c}} \\
5-1,6-8 \mathrm{c} \\
5-1,11-13\end{array}$ & $\begin{array}{r}6.10 \\
6.80 \\
8.66 \\
10.12 \\
31.54 \\
32.06 \\
34.56 \\
34.61\end{array}$ & $\begin{array}{l}\mathrm{pl}++, \mathrm{hb}+, \mathrm{au}+ \\
\mathrm{pl}++ \\
\mathrm{pl}++, \mathrm{hb}+ \\
\mathrm{pl}++, \mathrm{hb}+ \\
\mathrm{pl}+ \\
\mathrm{pl}++, \mathrm{hb}+, \mathrm{bi}+ \\
\mathrm{pl}++, \mathrm{gt}+, \mathrm{hb}+\end{array}$ & $\begin{array}{l}\mathrm{pm}>\mathrm{bw} \\
\mathrm{pm}>\mathrm{bw} \\
\mathrm{pm}>\mathrm{bw} \\
\mathrm{pm}>\mathrm{bw} \\
\mathrm{pm} \\
\mathrm{pm}<\mathrm{bw} \\
\mathrm{pm} \simeq \mathrm{bw} \\
\mathrm{pm} \simeq \mathrm{bw}\end{array}$ & $\begin{array}{l}\text { clear } \\
\text { clear } \\
\text { clear } \\
\text { clear } \\
\text { p. brown } \\
\text { clear } \\
\text { p. brown } \\
\text { p. brown }\end{array}$ & $\begin{array}{l}0.2 \\
0.15 \\
0.15 \\
0.2 \\
0.3 \\
0.5 \\
0.2 \\
0.3\end{array}$ & $\begin{array}{l}1.499-1.501 \\
1.499-1.501 \\
1.500-1.501 \\
1.500-1.501 \\
1.543-1.549 \\
1.500-1.501 \\
1.518-1.520 \\
1.499-1.518\end{array}$ & $1.500-1.504$ & $\begin{array}{l}1.500 \\
1.500 \\
1.5005 \\
1.5005 \\
1.546 \\
1.5005 \\
1.519 \\
1.502\end{array}$ \\
\hline
\end{tabular}


TABLE 2 - Continued

\begin{tabular}{|c|c|c|c|c|c|c|c|c|c|}
\hline \multirow[b]{2}{*}{ Age } & \multirow[b]{2}{*}{$\begin{array}{c}\text { Sample } \\
\text { (interval in } \mathrm{cm} \text { ) }\end{array}$} & \multirow{2}{*}{$\begin{array}{l}\text { Sub- } \\
\text { bottom } \\
\text { Depth } \\
\text { (m) }\end{array}$} & \multirow[b]{2}{*}{ Constituent Mineralsa } & \multirow[b]{2}{*}{ Typeb } & \multirow[b]{2}{*}{ Color } & \multicolumn{2}{|c|}{ Volcanic Glass } & \multirow[b]{2}{*}{ fractive Index } & \multirow[b]{2}{*}{ Mode } \\
\hline & & & & & & $\begin{array}{c}\text { Size } \\
(\mathrm{mm})\end{array}$ & Range & & \\
\hline Е్․․ & $444-5-4,34-36$ & 39.34 & $\mathrm{pl}++, \mathrm{op}+$ & $\mathrm{pm} \simeq \mathrm{bw}$ & clear & 0.8 & $1.500-1.501$ & & 1.5005 \\
\hline ¿ & $5-5,14-16$ & 40.64 & $\mathrm{pl}++, \mathrm{hb}+, \mathrm{hy}+$ & $\mathrm{pm}>$ bw & clear & 0.7 & $1.500-1.501$ & & 1.5005 \\
\hline$\underline{m}$ & $5-5,53-55$ & 41.03 & $\mathrm{pl}++, \mathrm{hb}+$ & $\mathrm{pm}>\mathrm{bw}$ & clear & 0.2 & $1.499-1.501$ & & 1.500 \\
\hline$\stackrel{\omega}{2}$ & & 42.18 & $\mathrm{pl}++$, hy + & $\mathrm{pm} \simeq \mathrm{bw}$ & clear & 0.2 & $1.508-1.513$ & & 1.511 \\
\hline \multirow{13}{*}{ 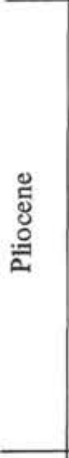 } & $444-7-2,19-21$ & 55.19 & $\mathrm{pl}+$ & $\mathrm{pm}>\mathrm{bw}$ & clear & 0.3 & $1.505-1.509$ & & 1.508 \\
\hline & $7-2,111-113$ & 56.11 & $\mathrm{pl}++, \mathrm{hb}+$ & $\mathrm{pm}>\mathrm{bw}$ & clear & 0.4 & $1.506-1.508$ & & 1.507 \\
\hline & $7-3,57-59$ & 57.07 & $\mathrm{pl}++, \mathrm{hb}+$ & $\mathrm{pm} \gg \mathrm{bw}$ & clear & 0.2 & $1.506-1.508$ & & 1.507 \\
\hline & $7-3,139-141$ & 57.89 & $\mathrm{pl}++, \mathrm{hy}+, \mathrm{hb}+$ & $\mathrm{pm}>$ bw & clear & 0.3 & $1.506-1.509$ & & 1.508 \\
\hline & $7-4,145-147$ & 59.45 & $\mathrm{pl}+$ & $\mathrm{pm} \gg \mathrm{bw}$ & clear & 0.1 & $1.506-1.509$ & & 1.508 \\
\hline & $7-5,80-82$ & 60.30 & $\mathrm{pl}+$, hy + & $\mathrm{pm}>\mathrm{bw}$ & clear & 0.13 & $1.506-1.509$ & & 1.508 \\
\hline & $7-6,17-19$ & 61.17 & $\mathrm{pl}++, \mathrm{hy}+, \mathrm{au}+$ & $\mathrm{pm}$ & p. brown & 0.2 & $1.523-1.530$ & $1.525-1.528$ & \\
\hline & $9-1,125-127 \mathrm{c}$ & 73.75 & $\mathrm{pl}++, \mathrm{hy}+, \mathrm{au}+, \mathrm{hb}+$ & $\mathrm{pm}>\mathrm{bw}$ & clear & 0.4 & $1.501-1.505$ & $1.502-1.504$ & \\
\hline & $10, \mathrm{CC}$ & 82.00 & $\mathrm{pl}+++, \mathrm{hy}, \mathrm{au}, \mathrm{op}, \mathrm{ol}, \mathrm{hb}+$ & $\mathrm{pm}$ & & & $1.510-1.520$ & & \\
\hline & $444 \mathrm{~A}-1-1,137-139$ & 83.37 & $\mathrm{pl}++, \mathrm{op}+, \mathrm{bi}+$ & $\mathrm{pm}>\mathrm{bw}$ & clear & 0.2 & $1.500-1.535$ & $1.505-1.515$ & \\
\hline & $1-3,10-12$ & 85.10 & $\mathrm{pl}+$ & $\mathrm{pm} \gg \mathrm{bw}$ & p. brown & 0.1 & $1.502-1.553$ & $1.515-1.535$ & \\
\hline & $1-3,38-40$ & 85.38 & $\mathrm{pl}+$ & $\mathrm{pm}>\mathrm{bw}$ & clear & 0.2 & $1.505-1.551$ & $1.510-1.535$ & \\
\hline & $1-5,44-46$ & 88.44 & $\mathrm{pl}+$ & $\mathrm{pm}>$ bw & clear & 0.25 & $1.505-1.510$ & & 1.507 \\
\hline \multirow{35}{*}{ 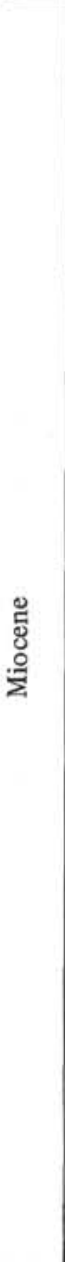 } & $2-2,72-74$ & 93.72 & $\mathrm{pl}+, \mathrm{op}+$ & $\mathrm{pm} \simeq \mathrm{bw}$ & clear & 0.45 & $1.505-1.509$ & & 1.507 \\
\hline & $2-2,115-117$ & 94.15 & $\mathrm{pl}+, \mathrm{hy}+, \mathrm{au}+$ & $\mathrm{pm}>\mathrm{bw}$ & $\begin{array}{l}\text { clear } \\
\text { p. brown }\end{array}$ & 0.5 & $1.505-1.509$ & & 1.507 \\
\hline & $2-3,36-38$ & 94.86 & $\mathrm{pl}+$ & $\mathrm{pm}>\mathrm{bw}$ & clear & 0.2 & $1.501-1.504$ & $1.502-1.504$ & 1.503 \\
\hline & $8-1,98-100$ & 149.48 & $\mathrm{pl}++$, hy,$+ \mathrm{au}+$, op + & $\mathrm{pm} \simeq \mathrm{bw}$ & $\begin{array}{l}\text { clear } \\
\text { p. brown }\end{array}$ & 0.4 & $1.506-1.510$ & & 1.507 \\
\hline & $9-1,115-117$ & 159.15 & $\mathrm{pl}+, \mathrm{au}+$ & sco & brown & 0.1 & $1.583-1.590$ & $1.585-1.589$ & \\
\hline & $9-2,7-9$ & 159.57 & $\mathrm{pl}+$, hy + & sco & brown & 1.5 & $1.585-1.590$ & & \\
\hline & $9-2,38-40$ & 159.88 & $\mathrm{pl}+, \mathrm{au}+$ & sco & p. brown & 0.7 & $1.552-1.583$ & & \\
\hline & $9-2,82-84$ & 160.32 & $\mathrm{pl}++, \mathrm{bi}, \mathrm{au}, \mathrm{hb}+$ & $\mathrm{pm} \simeq \mathrm{bw}$ & clear & 0.3 & $1.500-1.525$ & $1.500-1.510$ & \\
\hline & $9-2,112-114^{c}$ & 160.62 & $\mathrm{pl}++, \mathrm{au}+, \mathrm{hy}+$ & & brown & 0.5 & $1.528-1.578$ & & 1.577 \\
\hline & $9-3,70-72$ & 161.70 & $\mathrm{pl}++, \mathrm{au}+$ & sco & p. brown & 0.3 & $1.528-1.595$ & $1.585-1.590$ & \\
\hline & $9-4,17-19$ & 162.67 & $\mathrm{pl}+++$ & sco & brown & 0.7 & $1.510-1.590$ & & \\
\hline & $9-4,41-43$ & 162.91 & $\mathrm{pl}++, \mathrm{au}+$ & sco & brown & & $1.508-1.577$ & $1.520-1.570$ & \\
\hline & $9-4,86-88$ & 163.36 & $\mathrm{pl}++, \mathrm{au}+$ & sco & brown & 0.3 & $1.507-1.573$ & & \\
\hline & $9-5,5-7$ & 164.05 & $\mathrm{pl}++$, hy + & sco & brown & 0.3 & $1.540-1.585$ & $1.570-1.580$ & \\
\hline & $10-1,92-94^{c}$ & 168.42 & $\mathrm{pl}++, \mathrm{au}+$ & sco & brown & 0.2 & $1.580-1.615$ & $1.600-1.610$ & \\
\hline & $10-2,0-2$ & 169.00 & $\mathrm{pl}++, \mathrm{au}+$ & $\mathrm{pm}$ & p. brown & 0.1 & $1.525-1.532$ & & 1.528 \\
\hline & $10-2,30-32$ & 169.30 & $\mathrm{pl}+++$ & $\mathrm{pm}$ & $\begin{array}{l}\text { p. brown } \\
\text { clear }\end{array}$ & 1.0 & $1.523-1.531$ & & 1.528 \\
\hline & $10-2,114-116$ & 170.14 & $\mathrm{pl}++, \mathrm{au}+$ & $\mathrm{pm}$ & $\begin{array}{l}\text { p. brown } \\
\text { to clear }\end{array}$ & 0.8 & $1.510-1.550$ & $1.515-1.535$ & \\
\hline & $10-2,130-132$ & 170.30 & $\mathrm{pl}++$, hy + & $\mathrm{pm}$ & p. brown & 1.0 & $1.520-1.530$ & & \\
\hline & $11-2,68-70$ & 179.18 & $\mathrm{pl}++$, hy + & $\mathrm{pm}>\mathrm{bw}$ & clear & 0.25 & $1.509-1.514$ & $1.510-1.512$ & 1.511 \\
\hline & $11-3,83-85$ & 180.83 & $\mathrm{pl}++\mathrm{au}+$ & $\mathrm{pm}>$ bw & p. brown & 0.4 & $1.508-1.522$ & $1.509-1.514$ & \\
\hline & $12-1,59-61$ & 187.09 & $\mathrm{pl}++, \mathrm{hy}+, \mathrm{au}+$ & $\operatorname{sco}$ & $\begin{array}{l}\text { clear to } \\
\text { brown }\end{array}$ & 0.1 & $1.508-1.590$ & & \\
\hline & $12-1,119-121^{\mathrm{c}}$ & 187.69 & $\mathrm{pl}++, \mathrm{hy}+$ & & brown & 0.7 & $1.584-1.589$ & & 1.587 \\
\hline & $12-2,45-47 \mathrm{c}$ & 188.45 & & & p. brown & 0.4 & $1.584-1.588$ & & 1.586 \\
\hline & $13-2,0-2 \mathrm{c}$ & 197.50 & $\mathrm{pl}++, \mathrm{au}+$ & sco & brown & 0.2 & $1.531-1.583$ & $1.578-1.583$ & \\
\hline & $13-3,74-76^{c}$ & 199.74 & $\mathrm{pl}++, \mathrm{au}+$ & sco & brown & 0.3 & $1.575-1.590$ & & \\
\hline & $14-1,50-52$ & 206.00 & $\mathrm{pl}++, \mathrm{au}+$ & $\mathrm{pm} \gg \mathrm{bw}$ & p. brown & 0.3 & $1.526-1.535$ & $1.527-1.531$ & 1.528 \\
\hline & $14-2,30-32$ & 207.30 & & $\mathrm{pm}>\mathrm{bw}$ & p. brown & 0.4 & $1.519-1.530$ & $1.520-1.524$ & 1.523 \\
\hline & $14-4,40-42$ & 210.40 & $\mathrm{pl}+, \mathrm{au}+$ & $\mathrm{pm}>\mathrm{bw}$ & clear & 0.2 & $1.508-1.512$ & & 1.510 \\
\hline & $15-1,20-22$ & 215.20 & & $\mathrm{pm}>$ bw & clear & 0.4 & $1.507-1.510$ & & 1.508 \\
\hline & $15-1,97-99$ & 215.97 & $\mathrm{pl}++$, hy,+ au + & $\mathrm{pm}>\mathrm{bw}$ & clear & 0.3 & $1.507-1.510$ & & 1.509 \\
\hline & $15-2,77-79$ & 217.27 & $\mathrm{pl}++, \mathrm{au}+$ & $\mathrm{pm}>\mathrm{bw}$ & clear & 0.4 & $1.506-1.512$ & $1.507-1.510$ & 1.509 \\
\hline & $22-1,82-84^{c}$ & 282.32 & $\mathrm{pl}++$ & $\mathrm{pm}>$ bw & p. brown & 0.4 & $1.537-1.539$ & & 1.538 \\
\hline & $22-5,36-38$ & 287.86 & $\mathrm{pl}++, \mathrm{bi}+$ & $\mathrm{pm} \simeq \mathrm{bw}$ & clear & 0.3 & $1.497-1.500$ & & 1.498 \\
\hline & $22-5,58-60$ & 288.08 & $\mathrm{pl}++, \mathrm{au}+$ & $\mathrm{pm}>\mathrm{bw}$ & clear & 0.3 & $1.499-1.502$ & $1.500-1.501$ & 1.5005 \\
\hline
\end{tabular}

${ }^{\mathrm{a}} \mathrm{pl}=$ plagioclase, $\mathrm{au}=$ augite, $\mathrm{hy}=$ hypersthene, $\mathrm{hb}=$ hornblende, $\mathrm{bi}=$ biotite, $\mathrm{ol}=$ olivine, $\mathrm{op}=$ opaque minerals, $+++=$ very abundant, $++=$ abundant,$+=$ common .

$\mathrm{pm}=$ pumice type, $\mathrm{bw}=$ bubble-wall type, $\mathrm{sco}=$ scoria type.

${ }^{\mathrm{p}}$ Chemical analysis in Table 3. 

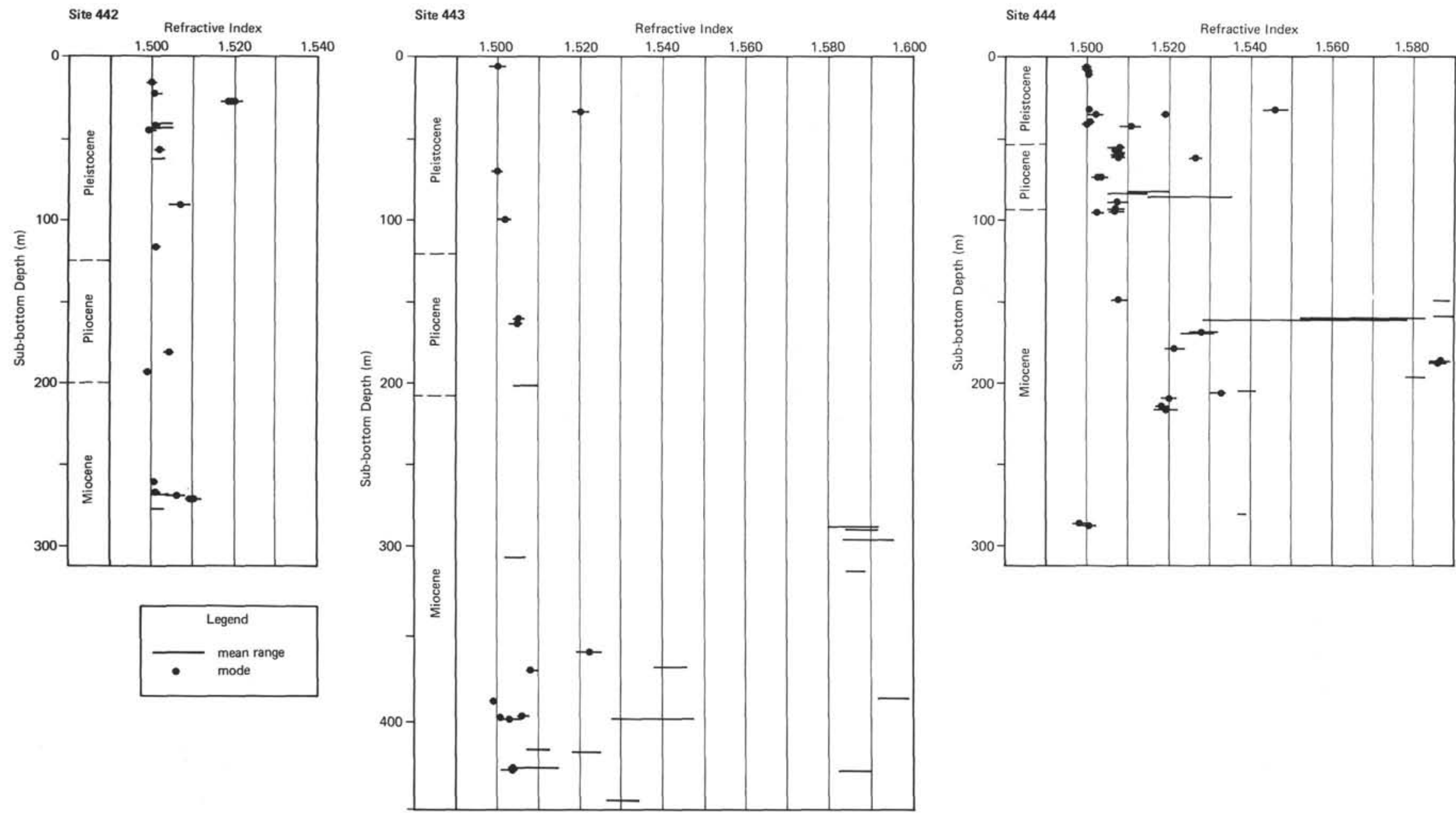

Figure 2. Refractive index of volcanic glass shards. 
TABLE 3

Chemical Composition of Volcanic Glass, Sites 442, 443, and 444

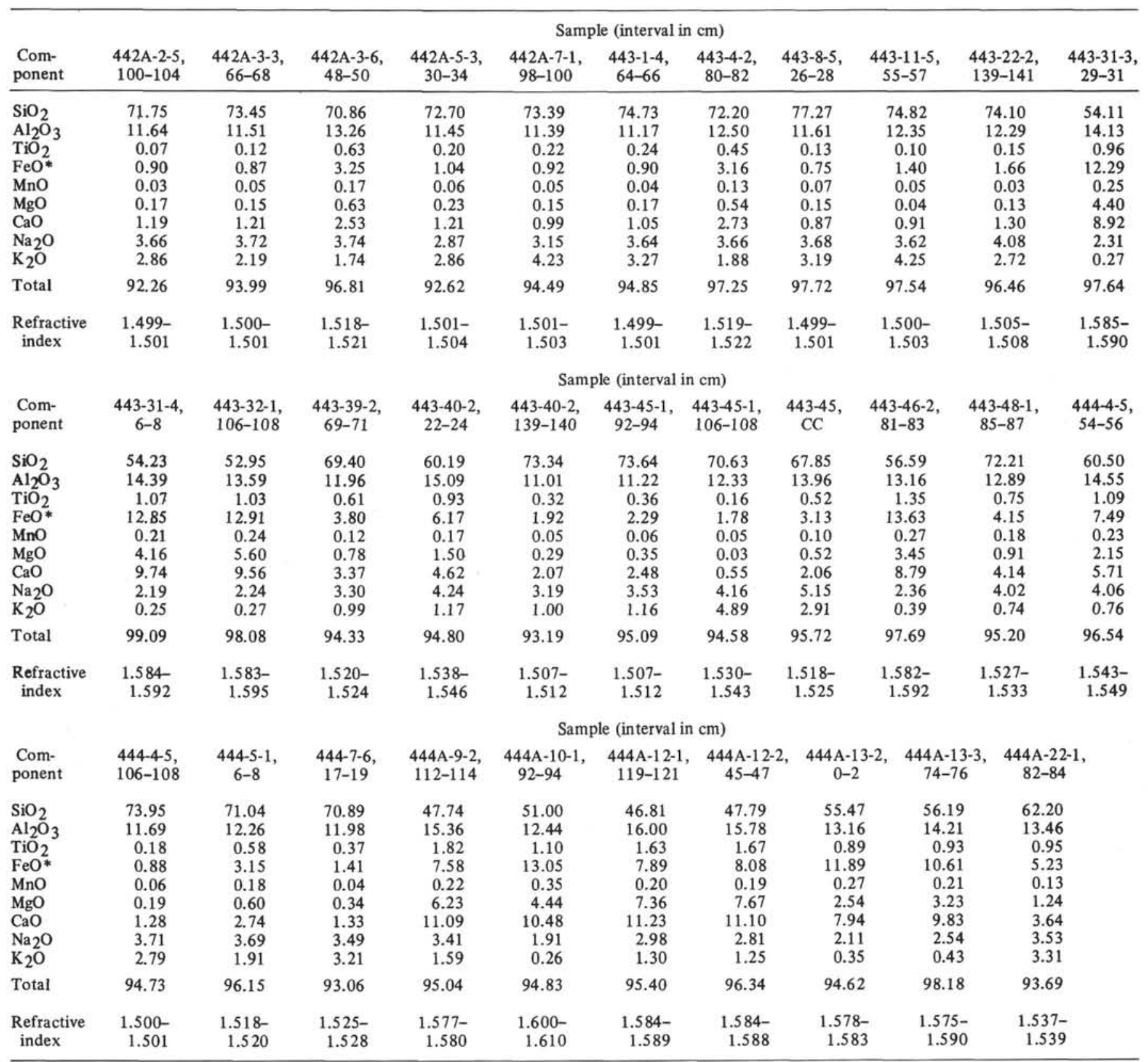

${ }^{*}$ Total iron as $\mathrm{FeO}$.

\section{RELATIONSHIP BETWEEN REFRACTIVE INDEX AND CHEMICAL COMPOSITION OF GLASS SHARDS}

According to Williams et al. (1955), $\mathrm{SiO}_{2}$ content of glass from artificial fusion of volcanic rocks and that of natural glass can be estimated from the refractive indexes. Recently, many investigators have reported the relationship between refractive index and $\mathrm{SiO}_{2}$ content: it is well known that as $\mathrm{SiO}_{2}$ content increases, refractive index decreases, although the relationship between the two parameters has not been established in detail yet.

In Figure 5, the mean ranges of refractive indexes from individual tephra layers (the range within which 80 per cent of the measurements fall) are plotted against average $\mathrm{SiO}_{2}$ content. The correlation between refractive index and $\mathrm{SiO}_{2}$ content is consistent, as a whole, with the trend given by Williams et al. (1955). In Figure 6 , the mean ranges of refractive indexes are plotted against the mean of total iron content of glass shards. The relation between refractive index and $\mathrm{FeO}$ content is quite linear. Because the refractivity of the transitionmetal oxides is higher than that of $\mathrm{SiO}_{2}$ and other oxides, the refractive index seems to be affected primarily by the content of the transition-metal oxides, such as $\mathrm{FeO}, \mathrm{TiO}_{2}$, and $\mathrm{MnO}$. In volcanic glass shards, $\mathrm{FeO}$ (or $\mathrm{Fe}_{2} \mathrm{O}_{3}$ ) predominates over the other transition-metal oxides, so that the refractive index of volcanic glass shards 

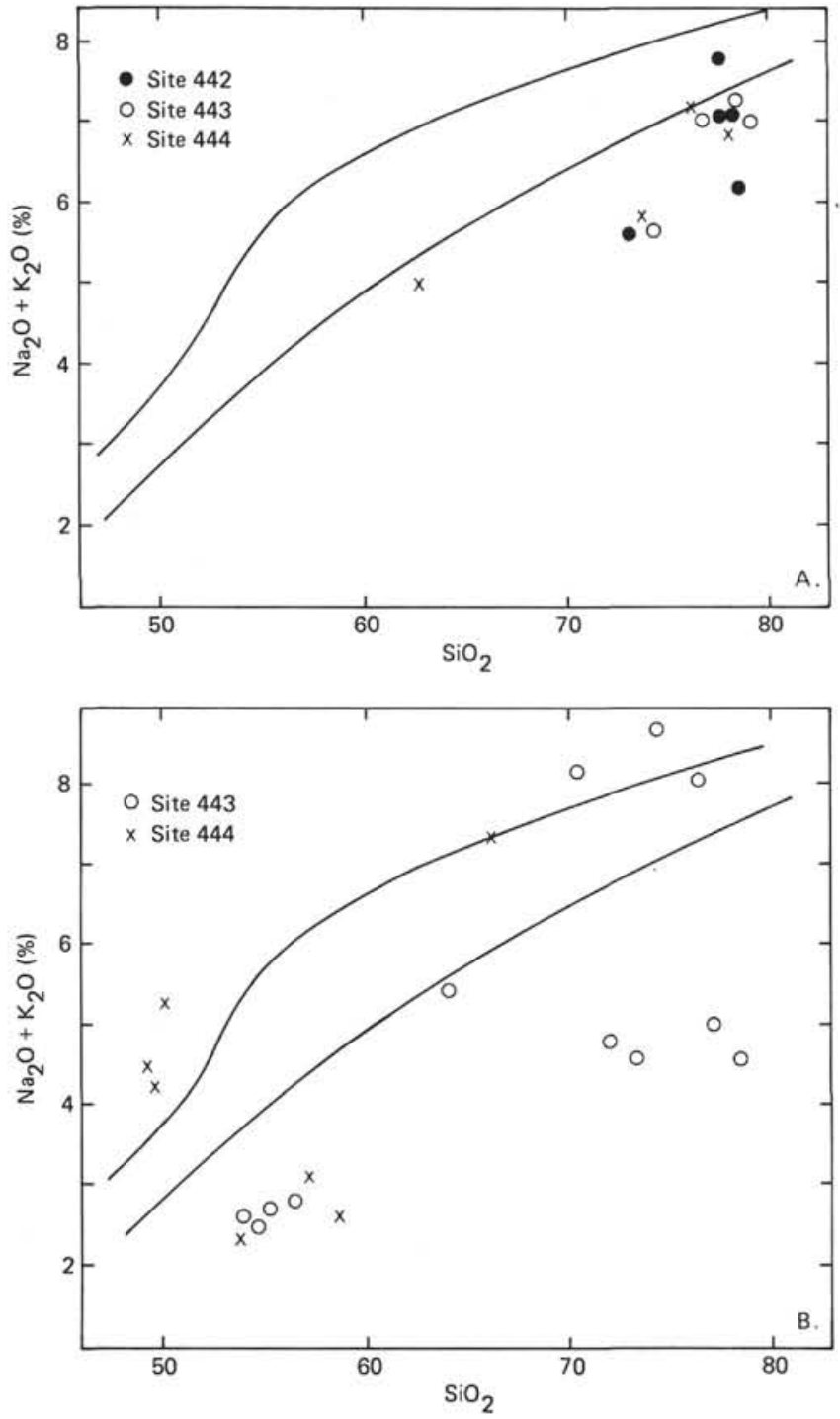

Figure 3. Total alkalis-SiO, relation for glass shards of tephra layers from the Shikoku Basin. A. Pleistocene and Pliocene tephra layers. B. Miocene tephra layers.

The two lines mark the general boundaries between the fields of the tholeiitic series, the high-alumina series, and the alkali series (Kuno, 1965).

can be determined by the content of the iron oxides alone.

The refractivity of ferric oxide is about 1.6 times greater than that of ferrous oxide, so that as ferric-oxide increases over ferrous oxide, the refractive index of glass shards increases. In Figure 6, some of the plotted data are off the general trend. In these exceptional samples, the ferric-iron content may be much higher than in glass which plots on the trend, because the total alkali- $-\mathrm{SiO}_{2}$ relation of these samples show that they belong to the alkali rock series.

\section{INTERPRETATIONS}

On the basis of petrographic characteristics, chemical composition, and paleontological age, the tephra layers from the Shikoku Basin are divided into the following groups: (1) non-alkali rhyolitic tephra layers in the Pleistocene and Pliocene at Sites 442, 443, and 444; (2) non-alkali rhyolitic tephra layers in the Miocene at Site 444; (3) non-alkali tholeiitic basalt tephra layers in the Miocene at Sites 443 and 444; (4) alkali rhyolitic tephra layers in the Miocene at Site 443; and (5) alkali basaltic tephra layers in the Miocene at Site 444.

It is clear that most Pleistocene and Pliocene tephra layers originated from non-alkali volcanism, whereas Miocene tephra layers originated from two different, simultaneous volcanic activities, as described below.

\section{Hydration of Glass Shards}

Hydration of volcanic glass shards has been discussed by many investigators (Aramaki and Lipman, 1965; Aramaki and Haramura, 1966; Jezek and Noble, 1978; Scheidegger et al., 1978; Ninkovich, 1979). Glass shards in deep-sea tephra layers are commonly subjected to hydration after deposition (Scheidegger et al., 1978; Ninkovich, 1979). In this study, glass shards of tephra layers from three sites seem to be hydrated, but visible alteration or hydrated rims of glass shards cannot be detected by optical observation. Electron-microprobe analysis of glass shards shows that most shards with the chemical composition of rhyolite and basalt are homogeneous from rim to core. In a few samples, shards are inhomogeneous: $\mathrm{Na}_{2} \mathrm{O}$ and $\mathrm{K}_{2} \mathrm{O}$ increase slightly from rim to core. In the present work, we omitted these samples.

\section{Relation Between Refractive Index and Some Oxides}

As shown by Williams et al. (1955), there is a close correlation between refractive index and $\mathrm{SiO}_{2}$ content in volcanic glass shards. This relation is summarized as follows: (1) with a $\mathrm{SiO}_{2}$ content of 72 to 73 per cent, the refractive index is about $1.50 ;(2)$ as the $\mathrm{SiO}_{2}$ content decreases, the refractive index increases proportionately, but the trend is slightly curved at $\mathrm{SiO}_{2}$ contents between 55 and 65 per cent. The same correlation between refractive index and $\mathrm{SiO}_{2}$ content of glass shards is established for deep-sea tephras in the Shikoku Basin.

The correlation between refractive index and total iron oxide is shown in Figure 6. This linear relationship is better than that between refractive index and $\mathrm{SiO}_{2}$ content.

The refractive index of volcanic glass shards primarily depends upon the contents of transition-metal oxides, because the refractivity of transition-metal oxides is higher than that of $\mathrm{SiO}_{2}$ and other chief constituent oxides (Larsen and Berman, 1943). Because the predominant constituent of transition-metal oxides is usually iron oxide, refractive index of glass shards is closely correlated to total iron. Although $\mathrm{TiO}_{2}$ has the highest refractivity among the transition-metal oxides in glass, its content is considerably smaller than that of iron oxide. In Figure 6, some data plot off the trend of correlation. The $\mathrm{TiO}_{2}$ content in these glass shards is larger than that in glass shards with a normal refractive index. If the total content of transition metal oxides in glass is plotted 

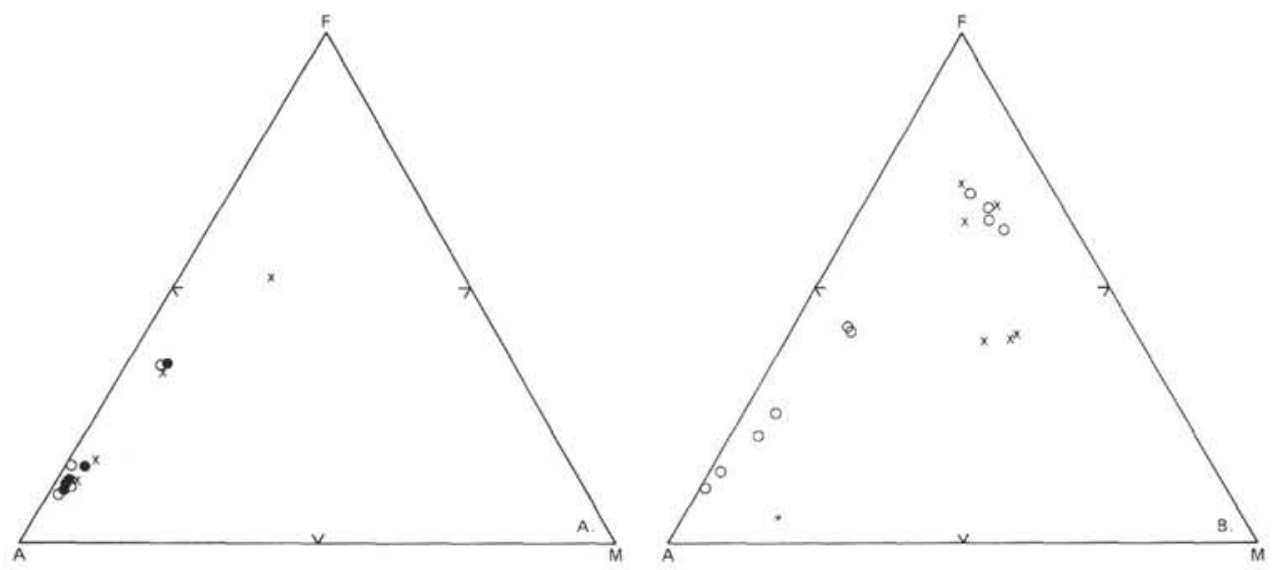

Figure 4. AFM (total iron-total alkali-MgO) diagram, showing the fractionation trend from basaltic to rhyolitic glass shards of tephra layers from the Shikoku Basin. Symbols as in Figure 3.

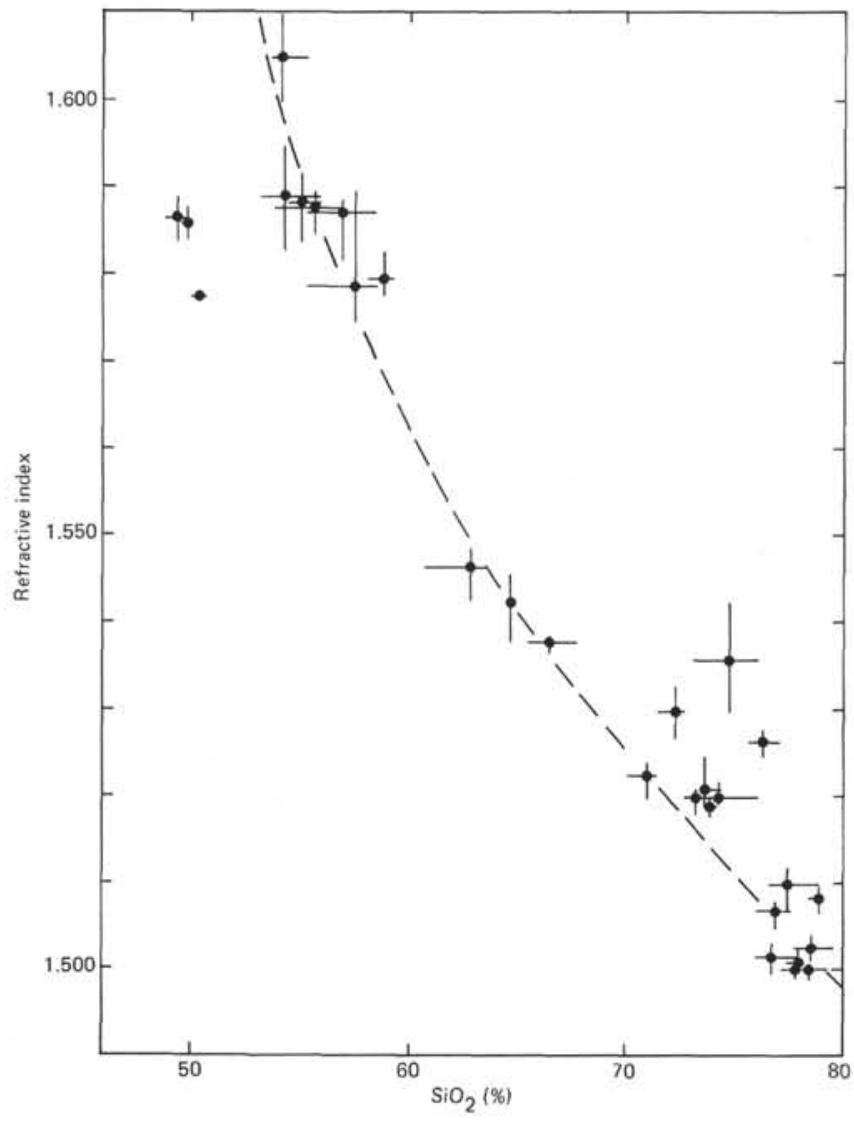

Figure 5. Relationship between refractive index and $\mathrm{SiO}_{2}$ content of volcanic glass shards. Horizontal bars denote the ranges of $\mathrm{SiO}_{2}$ content, and vertical bars denote the mean ranges of refractive index. Data are recalculated on a water-free basis.

against the refractive index, the correlation will be better. Another cause of this deviation seems to be the relatively large amount of ferric iron.

\section{Origins of the Deep-Sea Tephra Layers}

As noted, it is clear that the tephra layers from the central part of the Shikoku Basin have been derived

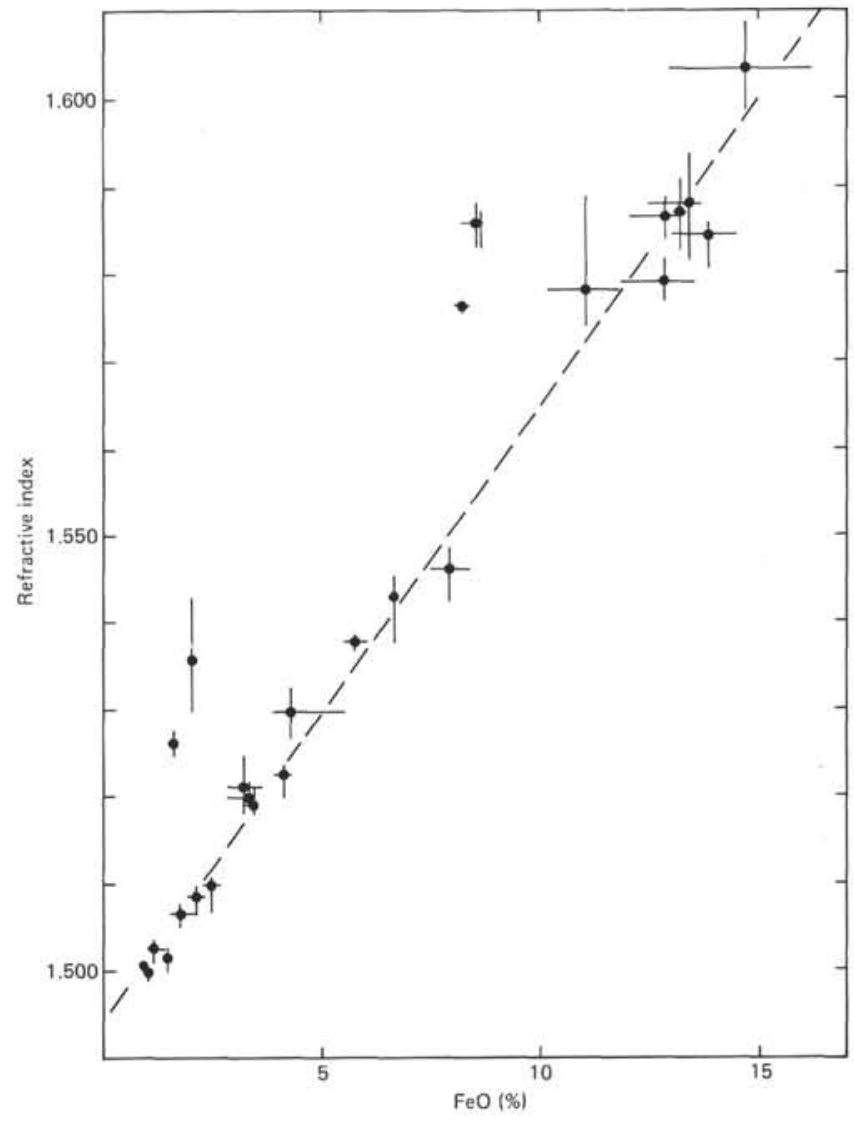

Figure 6. Relationship between refractive index and total iron oxide content. Horizontal bars denote the ranges of total iron oxide contents, and vertical bars denote the mean ranges of refractive index. Data are recalculated on a water-free basis.

from different volcanic sources with different rock series. Most of the Pleistocene and Pliocene tephra layers in this region are rhyolitic to dacitic (non-alkali series). These tephra layers presumably are ejecta produced by relatively large-scale eruptions in an island arc and transported toward the east: the grain size of shards in a well-correlated late-Pleistocene layer (442A-3-6, 48-50 
$\mathrm{cm} ; 443-4-3,80-82 \mathrm{~cm} ; 444-5-1,6-8 \mathrm{~cm})$ is greater in the westernmost site (442) than at the two eastern sites (443 and 444).

In contrast, the Miocene tephra layers are from rhyolitic to basaltic and belong to both the non-alkali and alkali rock series. In these tephras, the grain size of basaltic glass shards is usually greater than that of rhyolitic shards. This implies that sources of basaltic tephra were closer to the drilling sites than those of rhyolitic and andesitic tephra. Moreover, it is well known that the eruption columns of basaltic volcanoes are much smaller than those of felsic volcanoes, and the altitude attained by the ejecta is much lower. This results in much shorter distances of transport for basaltic ash falls, as compared with felsic ash falls. Therefore, the source of these basaltic tephra layers is assumed to have been close to the drilling sites.

A pillow basalt was dredged from one of the seamounts of the Kinan seamount chain in the central part of the Shikoku Basin; it was identified as tholeiitic basalt of late-Miocene age (Tokuyama and Fujioka, 1976). Petrographic study of basement basalts from the Shikoku Basin DSDP sites indicates that most are abyssal tholeiite, but a few sills belong to the alkali series (Dick et al., this volume). Tephra with high total alkali may be from such alkali volcanism. Another possible source is the Izu-Shichito-Iwo Jima volcanic arc, east of the drilling sites, although its history is not well understood. The activity of this arc probably ranges from the early Miocene to the Holocene (Kaneoka et al., 1970).

\section{ACKNOWLEDGMENTS}

We are very grateful to S. Aramaki, K. Kobayashi, and K. Fujioka for valuable suggestions and critical reading of the manuscript.

\section{REFERENCES}

Aramaki, S., and Lipman, P., 1965. Possible leaching of $\mathrm{Na}_{2} \mathrm{O}$ during hydration of volcanic glasses. Proc. Jap. Acad., 41, 467-470.
Aramaki, S., and Haramura, H., 1966. Leaching of $\mathrm{Na}_{2} \mathrm{O}$ from volcanic glasses during hydration-an experimental study. J. Geol. Soc. Japan, 72, 69-73.

Fujioka, K., Furuta, T., and Arai, F., in press. Petrography and geochemistry of volcanic glass of Leg 57. In von Huene, R., Nasu, N., et al., Init. Repts. DSDP, 56,57, Pt. 2: Washington (U.S. Govt. Printing Office).

Furuta, T., and Arai, F., in press. Petrographic properties of tephras in DSDP core from Leg 56. In Langseth, M., Okada, H., et al., Init. Repts. DSDP, 56, 57, Pt. 2: Washington (U.S. Govt. Printing Office).

Jezek, P. A., and Noble, D. C., 1978. Natural hydration and ion exchanges of obsidian: an electron microprobe study. Am. Mineral., 63, 266-273.

Jones, E. J. W., 1973. Volcanic glass in abyssal clays sampled at DSDP Leg 20 Drilling site, Northwest Pacific. In Heezen, B., MacGregor, I., et al., Init. Repts. DSDP, 20: Washington (U.S. Govt. Printing Office), pp. 389-416.

Kaneoka, I., Ishiki, N., and Zashu, S., 1970. K-Ar ages of the Izu-Bonin islands. Geochem. J., 4, 53-60.

Kuno, H., 1965. Fractionation trend of basalt magmas in lava flows. J. Petrol., 6, 302-321.

Larsen, E. R., and Berman, H., 1943. The microscopic determination of the nonopaque minerals. Bull. U.S. Geol. Surv., 843, 30-32.

Ninkovich, D., 1979. Distribution, age and chemical composition of tephra layers in deep-sea sediments off western Indonesia. J. Volcanol. Geotherm. Res., 5, 67-86.

Sheidegger, K. F., and Kulm, L. D., 1975. Late Cenozoic volcanism in the Aleutian Arcs: information from ash layers in the northeastern Gulf of Alaska. Geol. Soc. Am. Bull., 86, 1407-1412.

Sheidegger, K. F., Jezek, P. A., and Ninkovich, D., 1978. Chemical and optical studies of glass shards in Pleistocene and Pliocene ash layers from DSDP Site 192, northwestern Pacific Ocean. J. Volcanol. Geotherm. Res., 4, 99-116.

Tokuyama, H., and Fujioka, K., 1976. The petrologic study on basalt from Kinan Seamount and DSDP Site 54. Mar. Sci., 8, 184-191.

Williams, H., Turner, F. J., and Gilbert, C. M., 1955. Petrography-an Introduction to the Study of Rocks in Thin Sections: San Francisco (Freeman). 
APPENDIX

Petrographic Characteristics, Site 445 Tephra Layers ${ }^{\mathrm{a}}$

\begin{tabular}{|c|c|c|c|c|c|c|c|c|}
\hline \multirow{3}{*}{$\begin{array}{c}\text { Sample } \\
\text { (interval in } \mathrm{cm} \text { ) }\end{array}$} & \multirow{3}{*}{$\begin{array}{l}\text { Sub- } \\
\text { bottom } \\
\text { Depth } \\
\text { (m) }\end{array}$} & \multirow[b]{3}{*}{ Constituent Minerals } & \multicolumn{6}{|c|}{ Volcanic Glass } \\
\hline & & & \multirow[b]{2}{*}{ Type } & \multirow[b]{2}{*}{ Color } & \multirow{2}{*}{$\begin{array}{l}\text { Size } \\
(\mathrm{mm})\end{array}$} & \multicolumn{3}{|c|}{ Refractive Index } \\
\hline & & & & & & Range & Mean Range & Mode \\
\hline $445-1-5,33-35$ & 6.33 & $\mathrm{pl}+$ & $\mathrm{pm}<\mathrm{bw}$ & clear & 0.2 & $1.500-1.512$ & $1.509-1.511$ & \\
\hline $2-3,48-50$ & 11.98 & $\mathrm{pl}++, \mathrm{hy}+, \mathrm{au}+, \mathrm{hb}+, \mathrm{op}+$ & $\mathrm{pm}<\mathrm{bw}$ & clear & 0.4 & $1.502-1.503$ & & \\
\hline $3-3,114-116$ & 22.14 & & bw & clear & 0.4 & $1.500-1.504$ & & \\
\hline $8-4,59-61$ & 70.59 & & bw & clear & 0.2 & $1.501-1.512$ & & \\
\hline $10-5,90-92$ & 91.40 & $\mathrm{pl}++, \mathrm{hy}+, \mathrm{au}+, \mathrm{op}+$ & $\mathrm{pm}>\mathrm{bw}$ & p. brown & 0.5 & $1.505-1.540$ & $1.518-1.530$ & \\
\hline $12-3,31-33$ & 106.81 & $\mathrm{pl}++, \mathrm{hb}+, \mathrm{hy}+, \mathrm{op}+$ & $\mathrm{pm}>\mathrm{bw}$ & clear & 0.1 & $1.508-1.512$ & & \\
\hline $12-4,105-107$ & 109.05 & $\mathrm{pl}++, \mathrm{hb}++, \mathrm{au}+, \mathrm{op}+$ & $\mathrm{pm}>$ bw & clear & 0.1 & $1.503-1.509$ & $1.503-1.505$ & \\
\hline $13-4,118-120$ & 118.68 & $\mathrm{pl}+, \mathrm{op}+$ & $\mathrm{pm}>$ bw & $\begin{array}{l}\text { clear to } \\
\text { p. brown }\end{array}$ & 0.2 & $1.515-1.550$ & $1.515-1.530$ & \\
\hline $23-2,53-55$ & 210.03 & $\mathrm{bi}++, \mathrm{pl}+$ & $\mathrm{pm} \gg$ bw & clear & 0.1 & $1.501-1.503$ & & 1.502 \\
\hline $23-3,9-11$ & 211.09 & $\mathrm{pl}+, \mathrm{hb}+$ & $\mathrm{pm}$ & clear & 0.1 & $1.504-1.505$ & & \\
\hline $26-1,99-101$ & 237.49 & $\mathrm{pl}++$, bi,++ hy,+ op + & $\mathrm{pm}>\mathrm{bw}$ & clear & 0.2 & $1.499-1.501$ & & 1.500 \\
\hline $32-2,61-63$ & 295.61 & $\mathrm{pl}++, \mathrm{hy}+, \mathrm{au}+, \mathrm{op}+$ & $\mathrm{pm}<\mathrm{bw}$ & p. brown & 0.3 & $1.506-1.521$ & $1.506-1.508$ & 1.507 \\
\hline $46-2,7-8$ & 428.07 & $\mathrm{pl}++$, hy $+, \mathrm{au}^{+}, \mathrm{op}+$ & pm & p. brown & 0.1 & $1.522-1.528$ & $1.523-1.525$ & \\
\hline $55-6,30-32$ & 518.30 & $\mathrm{pl}++, \mathrm{op}+$ & $\mathrm{pm}$ & p. brown & 0.2 & $1.520-1.540$ & & \\
\hline $56-2,144-146$ & 524.44 & $\mathrm{pl}++, \mathrm{hy}+, \mathrm{au}+$ & $\mathrm{pm}>\mathrm{bw}$ & $\begin{array}{l}\text { clear to } \\
\text { p. brown }\end{array}$ & 0.2 & $1.512-1.514$ & & 1.513 \\
\hline $57-7,40-42$ & 540.40 & $\mathrm{pl}++, \mathrm{bi}++, \mathrm{hy}+$ & $\mathrm{pm}>\mathrm{bw}$ & clear & 0.5 & $1.501-1.503$ & & 1.502 \\
\hline $62-1,110-112$ & 579.60 & $\mathrm{pl}++$ & $\mathrm{pm}>\mathrm{bw}$ & $\begin{array}{l}\text { clear to } \\
\text { p. brown }\end{array}$ & 0.1 & $1.504-1.506$ & & 1.505 \\
\hline $62-2,89-90$ & 580.89 & $\mathrm{pl}++, \mathrm{au}+$ & $\mathrm{pm}$ & clear & 0.1 & $1.508-1.515$ & $1.508-1.511$ & \\
\hline $64-4,92-93$ & 602.92 & $\mathrm{pl}+$ & $\mathrm{pm}>$ bw & p. brown & 0.2 & $1.518-1.521$ & & 1.520 \\
\hline $64-4,114-115$ & 603.14 & $\mathrm{pl}+$ & $\mathrm{pm}$ & p. brown & 0.1 & $1.517-1.558$ & $1.525-1.540$ & \\
\hline $64-6,76-77$ & 605.76 & $\mathrm{pl}++, \mathrm{au}+$, op + & $\mathrm{pm} \cong \mathrm{bw}$ & p. brown & 0.2 & $1.509-1.511$ & & 1.510 \\
\hline $64-6,78-79$ & 605.78 & $\mathrm{pl}++, \mathrm{au}++$, op + & $\mathrm{pm}<\mathrm{bw}$ & p. brown & 0.5 & $1.509-1.512$ & & 1.511 \\
\hline $66-4,144-146$ & 622.44 & $\mathrm{pl}++, \mathrm{hy}+, \mathrm{au}+, \mathrm{op}+$ & $\mathrm{pm} \gg$ bw & clear & 0.1 & $1.507-1.510$ & & 1.508 \\
\hline
\end{tabular}

${ }^{\mathrm{a}}$ Symbols as in Table 2 .

\section{PLATE 1}

Figure $1442-\mathrm{A}-3-6,48-50 \mathrm{~cm}$. Glasses are almost pumice type. Refractive index $=1.517$ to 1.522 .

Figure $2443-1-4,64-66 \mathrm{~cm}$. Glasses are bubble-wall type. Refractive index $=1.499$ to 1.501 .

Figure $3443-11-5,55-57 \mathrm{~cm}$. Clean pumice-type glasses are predominant in this sample. Refractive index ranges from 1.500 to 1.503 .

Figure $4 \quad 443-31-4,6-8 \mathrm{~cm}$. Glasses are brown scoria type. Refractive index $=1.584$ to 1.592. Dark-brown devitrified glasses have numerous inclusions.

Figure $5 \quad 443-45-1,92-94 \mathrm{~cm}$. Glasses are clear bubble-wall type. Refractive index $=1.507$ to 1.513 .

Figure $6 \quad 443-46-2,81-83 \mathrm{~cm}$. Glasses are brown scoria type and have acicular crystallites. Refractive index $=1.582$ to 1.589 .

Figure 7 444A-12-1, 119-121 cm. Glasses are flake-shaped brown shards. Refractive in$\operatorname{dex}=1.584$ to 1.588 .

Figure $8 \quad$ Closeup of shard with crystallites from sample shown in Figure 7. This glass shard includes many crystallites, including scopulite and globulite. 


\section{PLATE 1}

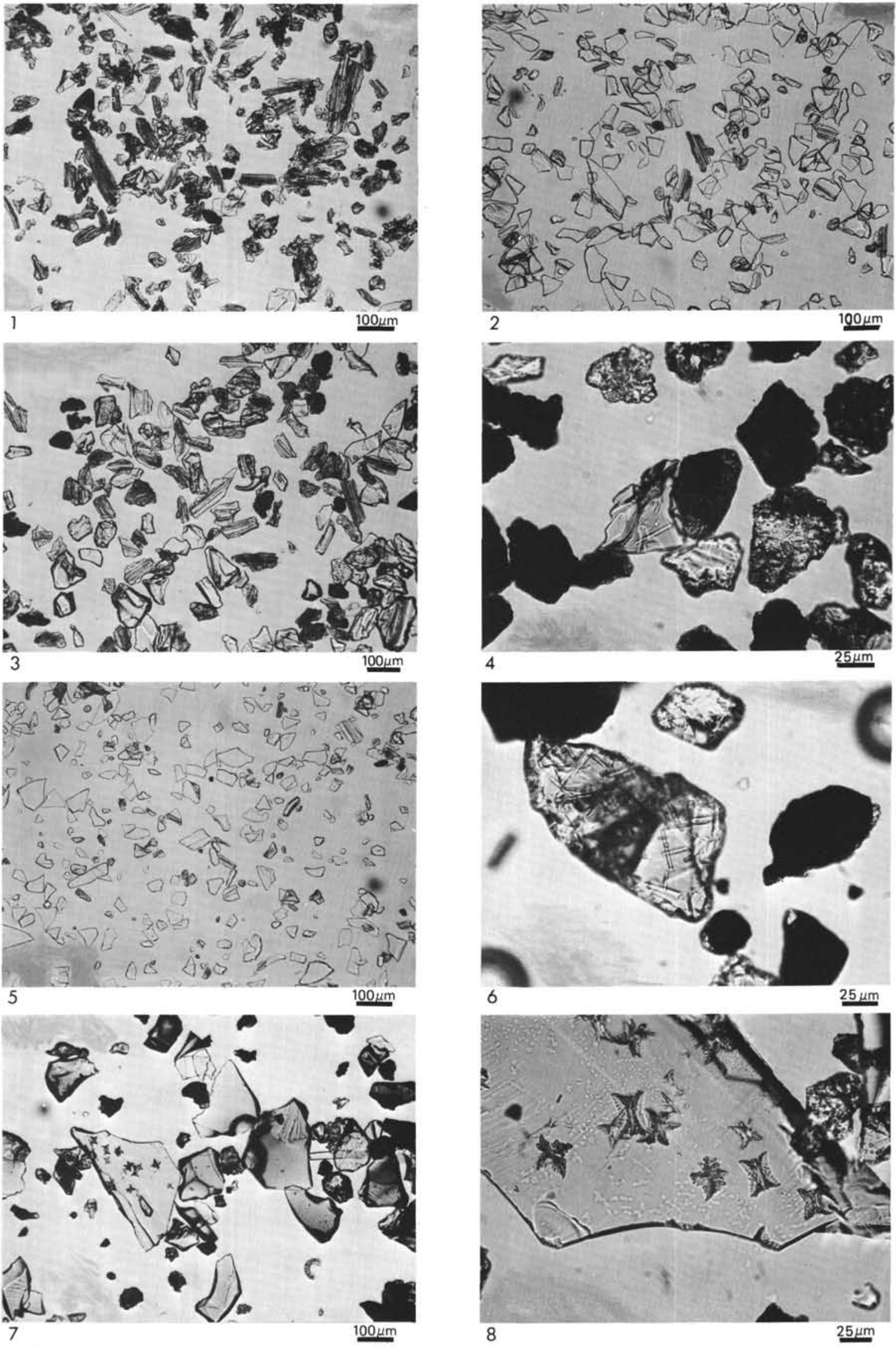

